\title{
Analytical Solutions of Functionally Graded Curved Beams under an Arbitrarily Directed Single Force
}

\author{
Liangliang Zhang, ${ }^{1,2}$ Lange Shang, ${ }^{1}$ and Yang Gao $\mathbb{D}^{1}$ \\ ${ }^{1}$ College of Science, China Agricultural University, Beijing 100083, China \\ ${ }^{2}$ Department of Civil Engineering and Engineering Mechanics, Columbia University, New York, NY 10027, USA \\ Correspondence should be addressed to Yang Gao; gaoyangg@gmail.com
}

Received 29 November 2018; Accepted 7 February 2019; Published 27 February 2019

Academic Editor: Gaetano Giunta

Copyright ( 2019 Liangliang Zhang et al. This is an open access article distributed under the Creative Commons Attribution License, which permits unrestricted use, distribution, and reproduction in any medium, provided the original work is properly cited.

\begin{abstract}
A functionally graded curved beam subjected to a shear tension force as well as a concentrated force at the free end is solved based on the inverse method, and a general two-dimensional solution is presented. The explicit expressions are derived by assuming that the elastic properties within curved beams vary in the radial direction according to a power law, i.e., $E=E_{0} r^{n}$, but are constant across the depth. After degenerating it into the isotropic homogeneous elastic cases, the results are in good consistency with existing analytical solutions. The stresses and displacements are firstly observed in different forms in terms of the different power function exponent $n$. These results will be useful as a guide for designing devices or as benchmark to assess other approximate methodologies.
\end{abstract}

\section{Introduction}

Functionally graded materials (FGMs) are inhomogeneous materials in which the volume fractions of two or more materials are varied continuously as a function of position along certain direction(s) of the structure to achieve required properties. Structures made of FGMs offer great application potential in engineering and industrial fields such as aerospace, automotive, petrochemical, and civil engineering fields [1-5]. Due to the increasing interest in FGMs in recent years, lots of studies have been performed on the static and dynamic behavior of functionally graded structures [6-12].

Curved beams are ones of frequently used components in frame structures, whose mechanical properties have been obtained by many researchers [13-16]. Many approximate methods, such as classical beam theory [17], Timoshenko's beam theory [18-21], and the high order beam theory [22], have been used to study the static displacement and stress distribution problems. For curved beams made of homogeneous materials, Timoshenko and Goodier [23] gave explicit solutions for curved beam under pure bending and curved cantilever beam under free end loads by using the Airy stress function method. Kilic and Aktas [24] got the elasticity solution for curved cantilever beams subjected to a single arbitrarily directed force at the free end. Bagci [25] studied the curved beams and rings of exponential thickness by using polar elasticity and state of plane stress. For functionally graded curved beams, Lekhnitskii [26] derived the solutions with elastic modulus being a product of a periodical function of the tangential coordinate and a power law function along the radial coordinate. Dryden [27] investigated the stress distribution of a functionally graded curved beam under pure bending where Young's modulus was in a more general form with proportional to $r^{2 \mathrm{~m}} \exp \left(\gamma r^{\mathrm{s}}\right)$ and Poisson's ratio was held constant. However, in his study, only pure bending problem is considered. Based on the Airy stress function method, Wang and Liu [28] extended Dryden's study [27] to the ones of the orthotropic functionally graded curved beams subjected to a uniform load on the outer surface and having various constraints or/and loads at ends. In his research, the beams are composed of $\mathrm{n}$ layers and each layer is orthotropic and functionally graded. Pydah and Sabale [29] analyzed the flexure of bidirectional functionally graded circular beams by using the kinematical assumptions of the Euler-Bernoulli theory. In the paper, the Young's modulus was graded along both radial direction and tangential direction. However, due to the mathematical complexity, they failed to carry out the 

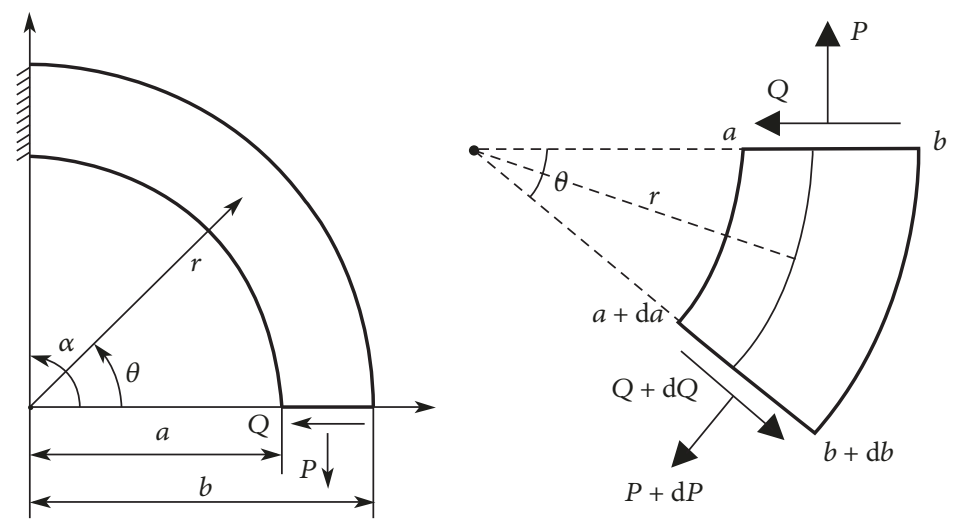

FIGURE 1: Sketch of functionally graded curved beam.

explicit formulation of the mechanical field. They also gave out a review of literature for functionally graded curved beams. By using the laminated model and the Taylor series expansion method, Shi el al. [30, 31] obtained the exact solutions of bending problem of a piezoelectric layered curved beam. Besides analytical studies, finite element methods play a significant role in the studied of functionally graded curved beams. De Miguel et al. [32] presented a new class of refined curved beam elements for the accurate stress analysis of composite structures. In their study, the kinematics of the beam element based on layer-wise and component-wise models and the locking-free curved beams elements based on the mixed interpolation of tensorial components method are provided. De Pietro et al. [33] investigated the mechanical behavior of slender and thick curved beams based on Naviertype analytical solution and a Carrera's unified formulation based weak form finite element solution.

In this paper, the elastic analysis for a functionally graded curved beam with Young's modulus varies as $E(r)=E_{0} r^{n}$ is carried out. The variation of exponent $n$ is considered, and the explicit expressions are derived based on the Airy stress function method. The stresses and the displacements are observed in different forms in terms of the different power function exponent $n$. These results will be useful as a guide for designing functionally graded devices or as benchmark to assess other approximate methodologies.

\section{Basic Equations}

Consider a functionally graded curved beam with unit depth referred to a polar coordinate system $(r, \theta)$. As shown in Figure 1 , the curved beam is bounded by inner and outer radii equal to $a$ and $b$ with the origin at the center of this curved beam and let $\theta=0$ and and $\theta=\alpha$ be the free end and the fixed end, respectively. Industrial loading applied on a curved beam could be decomposed to a moment and an arbitrarily directed concentrated force. As for pure bending problem, it was studied by Dryden [27]. In this paper, an arbitrarily directed single force was considered, which can be decomposed to a shear tension force $Q$ and a normal concentrated force $P$ applied on the free end as shown in Figure 1.
To start, the constitutive equations are

$$
\begin{aligned}
\varepsilon_{r} & =\frac{1}{E(r)}\left(\sigma_{r}-\nu \sigma_{\theta}\right), \\
\varepsilon_{\theta} & =\frac{1}{E(r)}\left(\sigma_{\theta}-\nu \sigma_{r}\right), \\
\gamma_{r \theta} & =\frac{1+\nu}{E(r)} \tau_{r \theta},
\end{aligned}
$$

where $E(r)=E_{0} r^{n}$ is the varying function of the Young's modulus, $E_{0}$ is the Young's modulus at $r=a$ of the curved beam, and Poisson's ratio $v$ is constant. Also, in (1), the shear modulus $G$ can be considered by letting $E(r)=2(1+v) G(r)$. The power function is either monotonically increasing or decreasing depending upon the index of the exponent $n$. As in previous treatments dealing with the analysis of inhomogeneous solids, Poisson's ratio is considered to be constant. Since the main interest is to find how the stiffness variation influences the flexural stress and displacement, this is not a serious deficiency.

The strain-displacement equations are

$$
\begin{aligned}
\varepsilon_{r} & =\frac{\partial u_{r}}{\partial r}, \\
\varepsilon_{\theta} & =\frac{1}{r} \frac{\partial u_{\theta}}{\partial \theta}+\frac{u_{r}}{r}, \\
\gamma_{r \theta} & =\frac{1}{2}\left(\frac{\partial u_{\theta}}{\partial r}+\frac{1}{r} \frac{\partial u_{r}}{\partial \theta}-\frac{u_{\theta}}{r}\right) .
\end{aligned}
$$

The strain compatibility equations are

$$
\begin{gathered}
\left(\frac{1}{r^{2}} \frac{\partial^{2}}{\partial \theta^{2}}-\frac{1}{r} \frac{\partial}{\partial r}\right) \varepsilon_{r}+\frac{1}{r^{2}} \frac{\partial}{\partial r}\left(r^{2} \frac{\partial \varepsilon_{\theta}}{\partial r}\right) \\
-\frac{2}{r^{2}} \frac{\partial}{\partial r}\left(r \frac{\partial \gamma_{r \theta}}{\partial \theta}\right)=0
\end{gathered}
$$


With a stress function $\varphi$, the stress expressions are

$$
\begin{aligned}
\sigma_{r} & =\frac{1}{r} \frac{\partial \varphi}{\partial r}+\frac{1}{r^{2}} \frac{\partial^{2} \varphi}{\partial \theta^{2}}, \\
\sigma_{\theta} & =\frac{\partial^{2} \varphi}{\partial r^{2}}, \\
\tau_{r \theta} & =-\frac{\partial}{\partial r}\left(\frac{1}{r} \frac{\partial \varphi}{\partial \theta}\right) .
\end{aligned}
$$

The boundary conditions are as follows:

$$
\begin{aligned}
r & =a, b: \\
\sigma_{r} & =\tau_{r \theta}=0, \\
\theta & =0: \\
\int_{a}^{b} \sigma_{\theta} \mathrm{d} r & =P, \\
\int_{a}^{b} \tau_{r \theta} \mathrm{d} r & =Q, \\
\int_{a}^{b}\left(r-\frac{a+b}{2}\right) \sigma_{\theta} \mathrm{d} r & =0 .
\end{aligned}
$$

The objective of this work is to present analytical solutions of functionally graded curved beams, assuming that the elastic properties vary in the radial direction according to a power law, i.e., $E=E_{0} r^{n}$, but are constant across the depth. In Sections 3 and 4, a shear tension force $Q$ and a normal concentrated force $P$ applied on the free end of functionally graded curved beams are analytically formulated in terms of Airy stress function. In Section 5, numerical results and discussion are provided for a specific example.

\section{Problem with a Shear Tension Force Q Applied}

Under a shear tension force $Q$, the stress function will relate to both $r$ and $\theta$. Here we assume that the stress function is $\varphi(r$, $\theta)=R(r) \sin \theta$ with the boundary conditions (5), where $Q \neq 0$ and $P=0$. Substituting $\varphi(r, \theta)$ into (1) then into (3), we obtain

$$
\begin{aligned}
r^{4} R^{(4)} & +2 r^{3}(1-n) R^{(3)}+r^{2}\left(n^{2}+\nu n-n-3\right) R^{\prime \prime} \\
& +r(3-v n)(1+n) R^{\prime}-(3-v n)(1+n)=0
\end{aligned}
$$

which satisfies the Euler equation with solution of $R=D r^{k}$. Substituting the solution into (6), we obtain

$$
\begin{aligned}
k^{4}- & (4+2 n) k^{3}+\left(n^{2}+v n+5 n+2\right) k^{2} \\
& -\left(n^{2}+v n^{2}+2 v n-4\right) k-(3-v n)(1+n)=0 .
\end{aligned}
$$

Then the characteristic roots of the aforementioned equation are $k_{0}=1$ and $k_{1}=1+n$, and the other two roots $k_{2}$ and $k_{3}$ must satisfy

$$
k^{2}-(2+n) k+v n-3=0 .
$$

Due to the values of $\nu$ which are $-1<v<1 / 2$, the roots $k_{2}$ and $k_{3}$ are different from each other. Considering the particular solutions of $n$, this problem can be divided into two cases, namely, Case 1: $n \neq-4 /(1-\nu)$ and Case $2: n=-4 /(1-\nu)$. In Case 1 , all roots are distinct to each other; in the Case 2, there are two pairs of repeated roots.

Case $1(n \neq-4 /(1-v))$. In this case, the stress function can be assumed as

$$
\varphi(r, \theta)=\left(D_{0} r+D_{i} r^{k_{i}}\right) \sin \theta
$$

where $D_{0}$ can be ignored for it has no effect on the stresses and displacements. In this paper, we take $i, j=1,2,3$. The summation convention has been used throughout this paper: the Einstein summation over repeated lower case indices is applied, while upper case indices take on the same numbers as the corresponding lower case ones but are not summed. By substituting $\varphi(r, \theta)$ into (4), the stresses can be derived

$$
\begin{aligned}
\sigma_{r} & =\sin \theta\left[D_{i}\left(k_{i}-1\right) r^{k_{I}-2}\right], \\
\sigma_{\theta} & =\sin \theta\left[D_{i} k_{i}\left(k_{I}-1\right) r^{k_{I}-2}\right], \\
\tau_{r \theta} & =-\cos \theta\left[D_{i}\left(k_{i}-1\right) r^{k_{I}-2}\right] .
\end{aligned}
$$

From the boundary conditions (5), we determined

$$
D_{i}=-\frac{\varepsilon_{i j k}\left(k_{J}-1\right)\left(k_{K}-1\right) a^{k_{j}} b^{k_{k}} Q}{\varepsilon_{i j k}\left(k_{I}-1\right)\left(k_{J}-k_{K}\right) a^{k_{i}} b^{k_{j}}\left(b^{k_{k}-1}-a^{k_{k}-1}\right)},
$$

where $\varepsilon_{i j k}$ is Levi-Civita symbol. The Levi-Civita symbol is defined as

$$
\begin{aligned}
& \varepsilon_{i j k} \\
& = \begin{cases}1, & \text { if }(i, j, k) \text { is an even permutation of }(1,2,3), \\
-1, & \text { if }(i, j, k) \text { is an odd permutation of }(1,2,3), \\
0, & \text { if } i=j \text { or } j=k \text { or } k=i .\end{cases}
\end{aligned}
$$

Substituting (10) into (1) and after integration, we can get the displacements as

$$
\begin{aligned}
u_{r}= & \frac{\sin \theta}{E_{0}}\left[D_{1} \ln r\left(n-v n-v n^{2}\right)+D_{2} r^{k_{2}-1-n}\right. \\
& \cdot \frac{\left(k_{2}-1+v k_{2}-v k_{2}^{2}\right)}{k_{2}-1-n}+D_{3} r^{k_{3}-1-n} \\
& \left.\cdot \frac{\left(k_{3}-1+v k_{3}-v k_{3}^{2}\right)}{k_{3}-1-n}\right]+f(\theta)
\end{aligned}
$$




$$
\begin{aligned}
u_{\theta} & =-\frac{\cos \theta}{E_{0}}\left\{D _ { 1 } \left[\left(n^{2}+n-v n\right)\right.\right. \\
& \left.-\left(n-v n-v n^{2}\right) \ln r\right]+D_{2} r^{k_{2}-1-n}\left(k_{2}-1\right) \\
& \left.\cdot \frac{k_{2}+v+2}{k_{2}-1-n}+D_{3} r^{k_{3}-1-n}\left(k_{3}-1\right) \frac{k_{3}+v+2}{k_{3}-1-n}\right\} \\
& -\int f(\theta) d \theta+g(r),
\end{aligned}
$$

where $f(\theta)$ and $g(r)$ are expressed as

$$
\begin{aligned}
f(\theta)= & A_{1} \cos \theta+A_{2} \sin \theta \\
& -\frac{D_{1}}{2 E_{0}} \theta \cos \theta\left(4 n+n^{2}-v n^{2}\right), \\
g(r)= & L r
\end{aligned}
$$

where $A_{1}, A_{2}$, and $L$ are arbitrary constants which can be determined from the constraint conditions of the curved beams. The corresponding displacements of $f(\theta)$ and $g(r)$ are $u_{r}{ }^{(0)}$ and $u_{\theta}{ }^{(0)}$, as follows:

$$
\begin{aligned}
u_{r}^{(0)}= & A_{1} \cos \theta+A_{2} \sin \theta \\
& -\frac{D_{1}\left(4 n+n^{2}-v n^{2}\right)}{2 E_{0}} \theta \cos \theta, \\
u_{\theta}^{(0)}= & -A_{1} \sin \theta+A_{2} \cos \theta+L r \\
& +\frac{D_{1}\left(4 n+n^{2}-v n^{2}\right)}{2 E_{0}}(\cos \theta+\theta \sin \theta) .
\end{aligned}
$$

In (15), it is shown that there are rigid body displacements in $u_{r}{ }^{(0)}$ and $u_{\theta}{ }^{(0)}$, which is $A_{1} \cos \theta+A_{2} \sin \theta$ in $u_{r}{ }^{(0)}$ and $-A_{1} \sin \theta+A_{2} \cos \theta+L r$ in $u_{\theta}{ }^{(0)}$. These two items are related to the translation and rotation of the rigid body, which can be balanced by applying a corresponding constraint. Thus, it can be ignored and then the displacements of the curved beam can be carried out as

$$
\begin{aligned}
u_{r}= & \frac{\sin \theta}{E_{0}}\left[D_{1} \ln r\left(n-v n-v n^{2}\right)+D_{2} r^{k_{2}-1-n}\right. \\
& \cdot \frac{\left(k_{2}-1+v k_{2}-v k_{2}^{2}\right)}{k_{2}-1-n}+D_{3} r^{k_{3}-1-n} \\
& \left.\cdot \frac{\left(k_{3}-1+v k_{3}-v k_{3}^{2}\right)}{k_{3}-1-n}\right]-\frac{D_{1}\left(4 n+n^{2}-v n^{2}\right)}{2 E_{0}} \theta
\end{aligned}
$$

$\cdot \cos \theta$

$$
\begin{aligned}
u_{\theta} & =-\frac{\cos \theta}{E_{0}}\left\{D _ { 1 } \left[\left(n^{2}+n-v n\right)\right.\right. \\
& \left.-\left(n-v n-v n^{2}\right) \ln r\right] \\
& +D_{2} r^{k_{2}-1-n}\left[\left(k_{2}-1\right) \frac{k_{2}+v+2}{k_{2}-1-n}\right] \\
& \left.+D_{3} r^{k_{3}-1-n}\left[\left(k_{3}-1\right) \frac{k_{3}+v+2}{k_{3}-1-n}\right]\right\} \\
& +\frac{D_{1}\left(4 n+n^{2}-v n^{2}\right)}{2 E_{0}}(\cos \theta+\theta \sin \theta) .
\end{aligned}
$$

Case $2(n=-4 /(1-\nu))$. In this case, we assume

$$
\varphi(r, \theta)=\left(D_{1} r^{1+n}+D_{2} r^{1+n} \ln r+D_{3} r \ln r\right) \sin \theta
$$

where $D_{i}$ are constants to be determined. Similarly, the stresses and displacements are summarized as follows:

$$
\begin{aligned}
& \sigma_{r}=\sin \theta\left(D_{1} n r^{n-1}+D_{2} r^{n-1}+D_{2} n r^{n-1} \ln r+D_{3} \frac{1}{r}\right) \\
& \sigma_{\theta}=\sin \theta\left[D_{1} n(1+n) r^{n-1}+D_{2}(1+2 n) r^{n-1}\right. \\
& \left.+D_{2} n(1+n) r^{n-1} \ln r+D_{3} \frac{1}{r}\right] \\
& \tau_{r \theta}=-\cos \theta\left(D_{1} n r^{n-1}+D_{2} r^{n-1}+D_{2} n r^{n-1} \ln r\right. \\
& \left.+D_{3} \frac{1}{r}\right) \text {. } \\
& u_{r}=\frac{\sin \theta}{E_{0}}\left[D_{1}\left(n-n v-v n^{2}\right) \ln r\right. \\
& +\frac{D_{2}}{2}\left(n-v n-v n^{2}\right)(\ln r)^{2} \\
& \left.+D_{2}(1-v-2 v n) \ln r-D_{3} \frac{1-v}{n} r^{-n}\right]+\frac{D_{2} n^{2}}{2 E_{0}} \theta \\
& \cdot \cos \theta \\
& u_{\theta}=\frac{\cos \theta}{E_{0}}\left[D_{1}\left(n-n v-v n^{2}\right) \ln r\right. \\
& -D_{1}\left(n^{2}+n-v n\right)+\frac{D_{2}}{2}\left(n-v n-v n^{2}\right)(\ln r)^{2} \\
& -\frac{D_{2}}{2}\left(n^{2}+2+4 n-2 v\right) \\
& +D_{2}\left(1-v-v n-n-n^{2}\right) \ln r \\
& \left.-D_{3} \frac{(1-v)(1+n)}{n} r^{-n}\right]-\frac{D_{2} n^{2}}{2 E_{0}} \theta \sin \theta \text {. }
\end{aligned}
$$




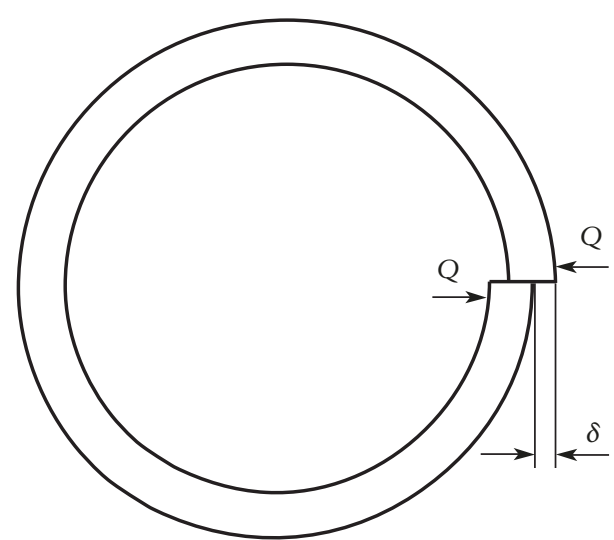

Figure 2: Edge dislocation of curved rings.

$$
\begin{aligned}
& D_{1}=\frac{Q\left(a^{n}-b^{n}+a^{n} n \ln a-b^{n} n \ln b\right)}{\left(a^{n}-b^{n}\right)^{2}-a^{n} b^{n} n^{2}(\ln a-\ln b)^{2}}, \\
& D_{2}=-\frac{Q n\left(a^{n}-b^{n}\right)}{\left(a^{n}-b^{n}\right)^{2}-a^{n} b^{n} n^{2}(\ln a-\ln b)^{2}}, \\
& D_{3}=-\frac{Q n^{2} a^{n} b^{n}(\ln a-\ln b)}{\left(a^{n}-b^{n}\right)^{2}-a^{n} b^{n} n^{2}(\ln a-\ln b)^{2}} .
\end{aligned}
$$

If we set $n=0$, then these results of Cases 1 and 2 can be degenerated to isotropic homogeneous elastic cases. According to (6), the characteristic roots $k_{0}, k_{1}, k_{2}$, and $k_{3}$ are $0,0,-1$, and 3 , respectively. As a result, we can assume the stress functions as

$$
\varphi(r, \theta)=\left(D_{0} r+D_{1} \frac{1}{r}+D_{2} r^{3}+D_{3} r \ln r\right) \sin \theta
$$

Thus in the similar way of Cases 1 and 2 by using this stress function, the solutions deduced here coincide well with existing solutions [17].

Assume the curved beam is expanded to be a curved ring by letting $\alpha=2 \pi$ as shown in Figure 2 . Considering $u_{r}$ in (16) and (19), there is an edge dislocation $\delta$ which is caused by the curved ring itself. To avoid this edge dislocation, we can add an additional shear tension force $Q$ on both the inner and the outer faces. By substituting $\delta=\left(u_{r}\right)_{\theta=2 \pi}-\left(u_{r}\right)_{\theta=0}$ into (11) and (20), the additional shear tension force $Q$ can be obtained for the two cases, respectively,

$$
\begin{aligned}
& Q_{2.1} \\
& =\delta E_{0} \frac{\varepsilon_{1 j k}\left(k_{1}-1\right)\left(k_{J}-k_{K}\right) a^{k_{1}} b^{k_{j}}\left(b^{k_{k}-1}-a^{k_{k}-1}\right)}{\varepsilon_{1 j k}\left(k_{J}-1\right)\left(k_{K}-1\right) a^{k_{j}} b^{k_{k}}\left(4 n+n^{2}-v n^{2}\right) \pi}, \\
& Q_{2.2}=-\frac{E_{0} \delta\left(\left(a^{n}-b^{n}\right)^{2}-a^{n} b^{n} n^{2}(\ln a-\ln b)^{2}\right)}{\pi n^{3}\left(a^{n}-b^{n}\right)} .
\end{aligned}
$$

\section{Problem with a Concentrated Force $P$ Applied}

This problem has the boundary conditions (5), where $P \neq 0$, $Q=0$. In the similar way of isotropic homogenous curved beams subjected to a concentrated force, we can assume the stress functions as $\varphi(r, \theta)=R_{0}(r)+R(r) \cos \theta$, in which $R_{0}(r)$ is only related to $r$. As a result, it is obvious to observe that $R_{0}(r)$ is the stress function of the pure bending problem which has been solved in our previous work [34] and $R(r) \cos \theta$ is the stress function of the problem solved in Section 3. For pure bending problem, the characteristic equation is

$$
\begin{gathered}
m^{4}-(4+2 n) m^{3}+\left(n^{2}+v n+5 n+4\right) m^{2} \\
-\left(n^{2}+v n^{2}+2 n+2 v n\right) m=0
\end{gathered}
$$

where the characteristic roots are $m_{0}=0$ and $m_{1}=2+n$, and the other two roots $m_{2}$ and $m_{3}$ satisfy

$$
m^{2}-(2+n) m+(1+v) n=0 .
$$

In virtue of $-1<v<1 / 2$, the roots $m_{2}$ and $m_{3}$ are different from each other. Considering the particular solutions of $n$, this problem can be divided into four cases, respectively, Case 3: $n \neq 1 / \nu, n \neq-4 /(1-\nu)$, and $n \neq-2$; all characteristic roots are distinct and $m_{i} \neq 1+n$; Case $4: n=1 / v$; all characteristic roots are distinct; without loss of generality, we take $m_{2}=1+n$ and $m_{3}=1$; Case 5: $n=-4 /(1-v)$; the characteristic roots $m_{i}$ are distinct and the roots $k_{i}$ have two pairs of repeated roots; Case 6: $n=-2$, the characteristic roots $m_{i}$ have a repeated root and the roots $k_{i}$ are distinct to each other. Combining with the cases which are given in Section 3, we can solve this problem in four different cases.

Case $3(n \neq 1 / \nu, n \neq-4 /(1-\nu)$ and $n \neq-2)$. In this case, all the characteristic roots are distinct to each other and $m_{i} \neq 1+n$. After the same manipulation as in Case 1, assume the stress functions as

$$
\begin{aligned}
R_{0}(r) & =C_{i} r^{m_{i}}, \\
R(r) & =D_{i} r^{k_{i}} .
\end{aligned}
$$

The stresses and the displacements without rigid body displacement in Case 3 can be stated as follows:

$$
\begin{aligned}
& \sigma_{r}=C_{i} m_{i} r^{m_{I}-2}+\cos \theta\left(D_{i}\left(k_{i}-1\right) r^{k_{I}-2}\right), \\
& \tau_{r \theta}=\sin \theta\left(D_{i}\left(k_{i}-1\right) r^{k_{I}-2}\right), \\
& \sigma_{\theta}=C_{i} m_{i}\left(m_{I}-1\right) r^{m_{I}-2}+\cos \theta\left(D_{i} k_{i}\left(k_{I}-1\right) r^{k_{I}-2}\right) .
\end{aligned}
$$




$$
\begin{aligned}
& u_{r}=\frac{\cos \theta}{E_{0}}\left[D_{1} \ln r\left(n-v n-v n^{2}\right)\right. \\
& +D_{2} r^{k_{2}-1-n} \frac{\left(k_{2}-1+v k_{2}-v k_{2}^{2}\right)}{k_{2}-1-n} \\
& \left.+D_{3} r^{k_{3}-1-n} \frac{\left(k_{3}-1+v k_{3}-v k_{3}^{2}\right)}{k_{3}-1-n}\right] \\
& +\frac{D_{1}\left(4 n+n^{2}-v n^{2}\right)}{2 E_{0}} \theta \sin \theta+C_{1} r \\
& \cdot \frac{(n+2)(1-v-v n)}{E_{0}}+C_{2} r^{m_{2}-n-1} \\
& \cdot \frac{m_{2}\left(1+v-v m_{2}\right)}{m_{2}-n-1}+C_{3} r^{m_{3}-n-1} \frac{m_{3}\left(1+v-\nu m_{3}\right)}{m_{3}-n-1} \text {, } \\
& u_{\theta}=\frac{\sin \theta}{E_{0}}\left[D_{1}\left(\left(n^{2}+n-v n\right)-\left(n-v n-v n^{2}\right) \ln r\right)\right. \\
& +D_{2} r^{k_{2}-1-n}\left(\left(k_{2}-1\right) \frac{k_{2}+v+2}{k_{2}-1-n}\right) \\
& \left.+D_{3} r^{k_{3}-1-n}\left(\left(k_{3}-1\right) \frac{k_{3}+v+2}{k_{3}-1-n}\right)\right] \\
& +\frac{D_{1}\left(4 n+n^{2}-v n^{2}\right)}{2 E_{0}}(-\sin \theta+\theta \cos \theta)+C_{1} r \theta \\
& \frac{(n+2)(n+v n)}{E_{0}} \\
& C_{i}=-\frac{\varepsilon_{i j k} m_{J} m_{K} a^{m_{j}} b^{m_{k}} P(a+b)}{2 \varepsilon_{i j k} m_{I} m_{J} a^{m_{i}} b^{m_{j}}\left(b^{m_{k}}-a^{m_{k}}\right)}, \\
& D_{i}=\frac{\varepsilon_{i j k}\left(k_{J}-1\right)\left(k_{K}-1\right) a^{k_{j}} b^{k_{k}} P}{\varepsilon_{i j k}\left(k_{I}-1\right)\left(k_{J}-k_{K}\right) a^{k_{i}} b^{k_{j}}\left(b^{k_{k}-1}-a^{k_{k}-1}\right)} .
\end{aligned}
$$

Case $4(n=1 / v)$. In this case, all the characteristic roots are distinct to each other. Without loss of generality, we take $m_{2}=$ $1+n$ and $m_{3}=1$. The stress function has the same expression as in Case 3. We can get that the stresses are the same as in (26), and the displacements in a similar manipulation are

$$
\begin{aligned}
u_{r}= & \frac{\cos \theta}{E_{0}}\left[D_{1} \ln r\left(n-\nu n-\nu n^{2}\right)+D_{2} r^{k_{2}-1-n}\right. \\
& \cdot \frac{\left(k_{2}-1+v k_{2}-v k_{2}^{2}\right)}{k_{2}-1-n}+D_{3} r^{k_{3}-1-n} \\
& \left.\cdot \frac{\left(k_{3}-1+v k_{3}-v k_{3}^{2}\right)}{k_{3}-1-n}\right]+\frac{D_{1}\left(4 n+n^{2}-v n^{2}\right)}{2 E_{0}} \theta \\
& \cdot \sin \theta-\frac{(2+n) v}{E_{0}} C_{1} r+\frac{m_{3}\left(1+v-v m_{3}\right)}{E_{0}\left(m_{3}-1-n\right)} \\
& \cdot C_{3} r^{m_{3}-1-n}+\frac{C_{2}(n+1)(n-v)}{E_{0}}
\end{aligned}
$$

$$
\begin{aligned}
u_{\theta} & =\frac{\sin \theta}{E_{0}}\left[D _ { 1 } \left(\left(n^{2}+n-v n\right)\right.\right. \\
& \left.-\left(n-v n-v n^{2}\right) \ln r\right)+D_{2} r^{k_{2}-1-n}\left(k_{2}-1\right) \\
& \left.\cdot \frac{k_{2}+v+2}{k_{2}-1-n}+D_{3} r^{k_{3}-1-n}\left(k_{3}-1\right) \frac{k_{3}+v+2}{k_{3}-1-n}\right] \\
& +\frac{D_{1}\left(4 n+n^{2}-v n^{2}\right)}{2 E_{0}}(-\sin \theta+\theta \cos \theta) \\
& +\frac{(2+n)(1+n)}{E_{0}} C_{1} r \theta,
\end{aligned}
$$

$C_{i}$ and $D_{i}$ are the same as in (28).

Case $5(n=-4 /(1-v))$. In this case, the characteristic roots $m_{i}$ are distinct to each other but the roots $k_{i}$ have two pairs of repeated roots. As a result, we assume

$$
\begin{aligned}
R_{0}(r) & =C_{i} r^{m_{i}}, \\
R(r) & =D_{1} r^{1+n}+D_{2} r^{1+n} \ln r+D_{3} r \ln r .
\end{aligned}
$$

Then the stresses and the displacements without rigid body displacement can be obtained as

$$
\begin{aligned}
& \sigma_{r}=\cos \theta\left(D_{1} n r^{n-1}+D_{2} r^{n-1}+D_{2} n r^{n-1} \ln r+D_{3} \frac{1}{r}\right) \\
& +C_{i} m_{i} r^{m_{I}-2} \\
& \sigma_{\theta}=\cos \theta\left[D_{1} n(1+n) r^{n-1}+D_{2}(1+2 n) r^{n-1}\right. \\
& \left.+D_{2} n(1+n) r^{n-1} \ln r+D_{3} \frac{1}{r}\right]+C_{i} m_{i}\left(m_{I}-1\right) \\
& \cdot r^{m_{I}-2} \\
& \tau_{r \theta}=\sin \theta\left(D_{1} n r^{n-1}+D_{2} r^{n-1}+D_{2} n r^{n-1} \ln r\right. \\
& \left.+D_{3} \frac{1}{r}\right) \text {. } \\
& u_{r}=\frac{1}{E_{0}}\left(C_{1} r(n+2)(1-v-v n)\right. \\
& +C_{2} r^{m_{2}-n-1} \frac{m_{2}\left(1+v-v m_{2}\right)}{m_{2}-n-1} \\
& \left.+C_{3} r^{m_{3}-n-1} \frac{m_{3}\left(1+v-v m_{3}\right)}{m_{3}-n-1}\right) \\
& +\frac{\cos \theta}{E_{0}}\left[D_{1}\left(n-n v-v n^{2}\right) \ln r\right. \\
& +D_{2}\left(n-v n-v n^{2}\right)(\ln r)^{2}+D_{2}(1-v-2 v n) \ln r \\
& \left.-D_{3} \frac{1-v}{n} r^{-n}\right]-\frac{D_{2} n^{2}}{2 E_{0}} \theta \sin \theta,
\end{aligned}
$$




$$
\begin{aligned}
& u_{\theta}=-\frac{\sin \theta}{E_{0}}\left[D_{1}\left(n-n v-v n^{2}\right) \ln r\right. \\
& -D_{1}\left(n^{2}+n-v n\right)+\frac{D_{2}}{2}\left(n-v n-v n^{2}\right)(\ln r)^{2} \\
& -\frac{D_{2}}{2}\left(n^{2}+2+4 n-2 \nu\right) \\
& +D_{2}\left(1-v-v n-n-n^{2}\right) \ln r \\
& \left.-D_{3} \frac{(1-\nu)(1+n)}{n} r^{-n}\right]-\frac{D_{2} n^{2}}{2 E_{0}} \theta \cos \theta+C_{1} r \theta \\
& \frac{(n+2)(n+v n)}{E_{0}} \\
& C_{i}=-\frac{\varepsilon_{i j k} m_{J} m_{K} a^{m_{j}} b^{m_{k}} P(a+b)}{2 \varepsilon_{i j k} m_{I} m_{J} a^{m_{i}} b^{m_{j}}\left(b^{m_{k}}-a^{m_{k}}\right)}, \\
& D_{1}=-\frac{P\left(a^{n}-b^{n}+a^{n} n \ln a-b^{n} n \ln b\right)}{\left(a^{n}-b^{n}\right)^{2}-a^{n} b^{n} n^{2}(\ln a-\ln b)^{2}}, \\
& D_{2}=\frac{\operatorname{Pn}\left(a^{n}-b^{n}\right)}{\left(a^{n}-b^{n}\right)^{2}-a^{n} b^{n} n^{2}(\ln a-\ln b)^{2}}, \\
& D_{3}=\frac{p n^{2} a^{n} b^{n}(\ln a-\ln b)}{\left(a^{n}-b^{n}\right)^{2}-a^{n} b^{n} n^{2}(\ln a-\ln b)^{2}} .
\end{aligned}
$$

Case $6(n=-2)$. In this case, the characteristic roots $m_{i}$ have a repeated root and the roots $k_{i}$ are distinct to each other. Thus, we assume

$$
\begin{aligned}
R_{0}(r) & =C_{1} \ln r+C_{2} r^{m_{2}}+C_{3} r^{m_{3}}, \\
R(r) & =D_{i} r^{k_{i}} .
\end{aligned}
$$

Then the stresses and the displacements without rigid body displacement can be obtained as

$$
\begin{aligned}
\sigma_{r}= & \frac{C_{1}}{r^{2}}+C_{2} m_{2} r^{m_{2}-2}+C_{3} m_{3} r^{m_{3}-2}+\cos \theta \\
\cdot & \left(D_{i}\left(k_{i}-1\right) r^{k_{I}-2}\right), \\
\sigma_{\theta}= & -\frac{C_{1}}{r^{2}}+C_{2} m_{2}\left(m_{2}-1\right) r^{m_{2}-2}+C_{3} m_{3}\left(m_{3}-1\right) \\
& \cdot r^{m_{3}-2}+\cos \theta\left(D_{i} k_{i}\left(k_{I}-1\right) r^{k_{I}-2}\right), \\
\tau_{r \theta} & =\sin \theta\left(D_{i}\left(k_{i}-1\right) r^{k_{I}-2}\right) .
\end{aligned}
$$

$$
\begin{aligned}
& u_{r}=\frac{1}{E_{0}}\left(-C_{1} r^{-1-n}(1+\nu)(1+n)\right. \\
& +C_{2} m_{2} \frac{1+v-v m_{2}}{m_{2}-n-1} r^{m_{2}-1-n} \\
& \left.+C_{3} m_{3} \frac{1+v-v m_{3}}{m_{3}-n-1} r^{m_{3}-1-n}\right) \\
& +\frac{\cos \theta}{E_{0}}\left[D_{1} \ln r\left(n-v n-v n^{2}\right)\right. \\
& +D_{2} r^{k_{2}-1-n} \frac{\left(k_{2}-1+v k_{2}-v k_{2}^{2}\right)}{k_{2}-1-n} \\
& \left.+D_{3} r^{k_{3}-1-n} \frac{\left(k_{3}-1+v k_{3}-v k_{3}^{2}\right)}{k_{3}-1-n}\right] \\
& +\frac{D_{1}\left(4 n+n^{2}-v n^{2}\right)}{2 E_{0}} \theta \sin \theta, \\
& u_{\theta}=\frac{\theta}{E_{0}}\left[C_{1} r^{-1-n}(\nu n+n)\right] \\
& +\frac{D_{1}\left(4 n+n^{2}-v n^{2}\right)}{2 E_{0}}(-\sin \theta+\theta \cos \theta) \\
& \cdot \frac{\sin \theta}{E_{0}}\left\{D_{2} r^{k_{2}-1-n}\left[\left(k_{2}-1\right) \frac{k_{2}+v+2}{k_{2}-1-n}\right]\right. \\
& +D_{3} r^{k_{3}-1-n}\left[\left(k_{3}-1\right) \frac{k_{3}+v+2}{k_{3}-1-n}\right] \\
& \left.+D_{1}\left(\left(n^{2}+n-v n\right)-\left(n-v n-v n^{2}\right) \ln r\right)\right\} . \\
& C_{1}=\frac{(a+b)\left(a^{m_{3}} b^{m_{2}}-a^{m_{2}} b^{m_{3}}\right) m_{2} m_{3} P}{D}, \\
& C_{2}=-\frac{(a+b)\left(a^{m_{3}}-b^{m_{3}}\right) m_{3} P}{D}, \\
& C_{3}=\frac{(a+b)\left(a^{m_{2}}-b^{m_{2}}\right) m_{2} P}{D} \\
& D_{i}=\frac{\varepsilon_{i j k}\left(k_{J}-1\right)\left(k_{K}-1\right) a^{k_{j}} b^{k_{k}} P}{\varepsilon_{i j k}\left(k_{I}-1\right)\left(k_{J}-k_{K}\right) a^{k_{i}} b^{k_{j}}\left(b^{k_{k}-1}-a^{k_{k}-1}\right)}, \\
& D=2\left[\left(a^{m_{3}}-b^{m_{3}}\right)\left(a^{m_{2}}-b^{m_{2}}\right)\left(m_{2}-m_{3}\right)\right. \\
& \left.-\left(a^{m_{3}} b^{m_{2}}-a^{m_{2}} b^{m_{3}}\right) m_{2} m_{3} \ln \frac{b}{a}\right] .
\end{aligned}
$$




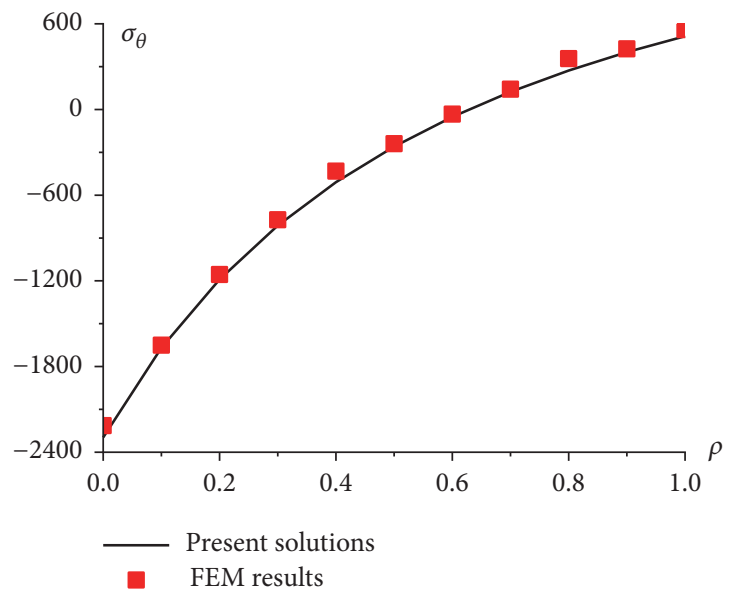

(a) $\rho=(r-a) / a$

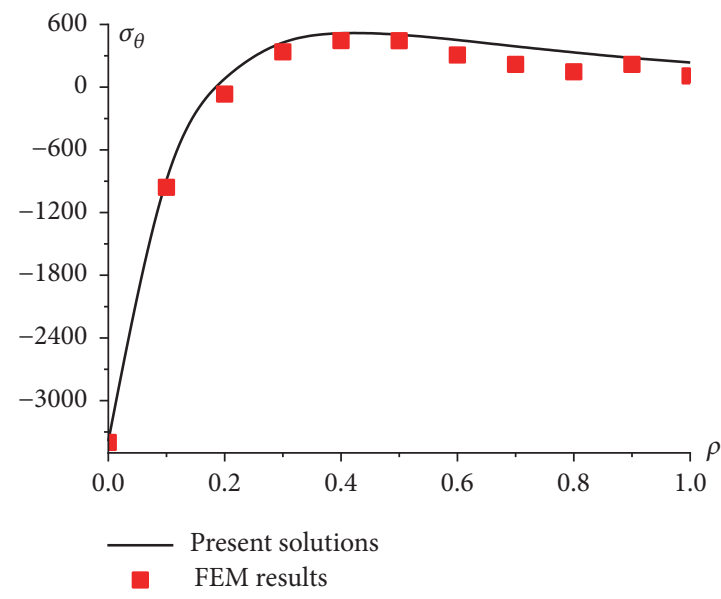

(b) $\rho=(r-a) / a$

FIGURE 3: Validation of the present solution by comparing with the finite element results: elastic curved beams $n=0$ (a) and functionally graded curved beams $n=2$ (b).

\section{Numerical Results and Discussion}

In this section, numerical studies and discussion will be presented. Before that, it is appropriate to verify the accuracy of the formulation. The validation studies are investigated by comparing the proposed solutions with finite element results for elastic and functionally graded curved beams. Specifically, we take SiC/C FGM as a specimen, whose $E_{0}$ is $400 \mathrm{GPa}$. The beam is set up as the same as Figure $1(a=6 \mathrm{~mm}, b$ $=12 \mathrm{~mm}$ ) subjected to a tension force $P=100 \mathrm{~N}$ at the free end $(\theta=0)$. For convenience, we take Poisson's ratio as 0.2. To conduct the finite element solution, commercial software ANSYS is used with the beam element BEAM188 and a mesh with 50 elements along the axis. The stress $\sigma_{\theta}$ at the section $\theta=\pi / 3$ is collected to make the comparisons which are shown in Figure 3 with elastic curved beams $n=0(a)$ and functionally graded curved beams $n=2(b)$. To avoid the size effect, we take $\rho=(r-a) / a$. It is worth mentioning that the present solutions show great agreement with the finite element results.

When it comes to numerical simulation, we consider the particular solutions of $n$; the graded exponent can be taken as $-5,-2,0,2$, and 5 . Figure 4 shows the variation of displacements $u_{r}$ and $u_{\theta}$ and stresses $\sigma_{r}, \sigma_{\theta}$, and $\tau_{r \theta}$ at the section $\theta=\pi / 3$ with coordinate $r$. To avoid the size effect, we take $\rho=(r-a) / a$.

It is observed that, in Figures 4(c) and 4(d), the displacement $u_{r}$ stays nearly uniform across the radius while the displacement $u_{\theta}$ varies nearly linearly with coordinate $\rho$. The distribution of stress $\sigma_{\theta}$ as shown in Figure 4(e) is nonlinear along the radius direction for FGM $(n \neq 0)$, different from the nearly linear distribution for a homogeneous material $(n=0)$. The variation of stress $\sigma_{r}$ which is exhibited in Figure 4(a) is very similar to $\tau_{r \theta}$, except that the values of whose are opposite. It is worthy to notice that the values of $\sigma_{\theta}$ are much bigger compared to $\sigma_{r}$ and $\tau_{r \theta}$ at the inner and outer faces. From Figures 4(a) and 4(b), it is expected to observe that the stresses $\sigma_{r}$ and $\tau_{r \theta}$ increase from zero at the inner face $(\rho=0)$ to the peak and then decrease to zero at the outer face $(\rho=1)$. And the maximum values of $\sigma_{r}$ and $\tau_{r \theta}$ are shifted from the inner face to the outer face. The stress and displacement field of the curved beam is greatly influenced by graded exponent $n$, which means that the curved beam can be optimally designed according to given working conditions by adjusting the graded material properties.

\section{Concluding Remarks}

The analytical elasticity solutions were derived for functionally graded curved beams by means of the inverse method. The mechanical properties of the material were assumed to be the same across the depth. Then a general two-dimensional solution was obtained for functionally graded curved beams subjected to a shear tension force $Q$ as well as a concentrated force $P$ at the free end and was validated with finite element results, which showed great agreement with the proposed formulation. In this study, we observed the power function exponent $n$ had strong effect on stresses and displacements. The expressions of the solutions were in different forms when the exponent $n$ was assigned a characteristic value. The obtained solution is valid for graded variations as a power law, i.e., $E=E_{0} r^{n}$, so it could serve as a guide for designing or as a benchmark to assess other approximate methodologies.

\section{Data Availability}

The figure data (Figures 3(a)-3(e)) used to support the findings of this study are included within the article.

\section{Conflicts of Interest}

The authors declare that they have no conflicts of interest. 

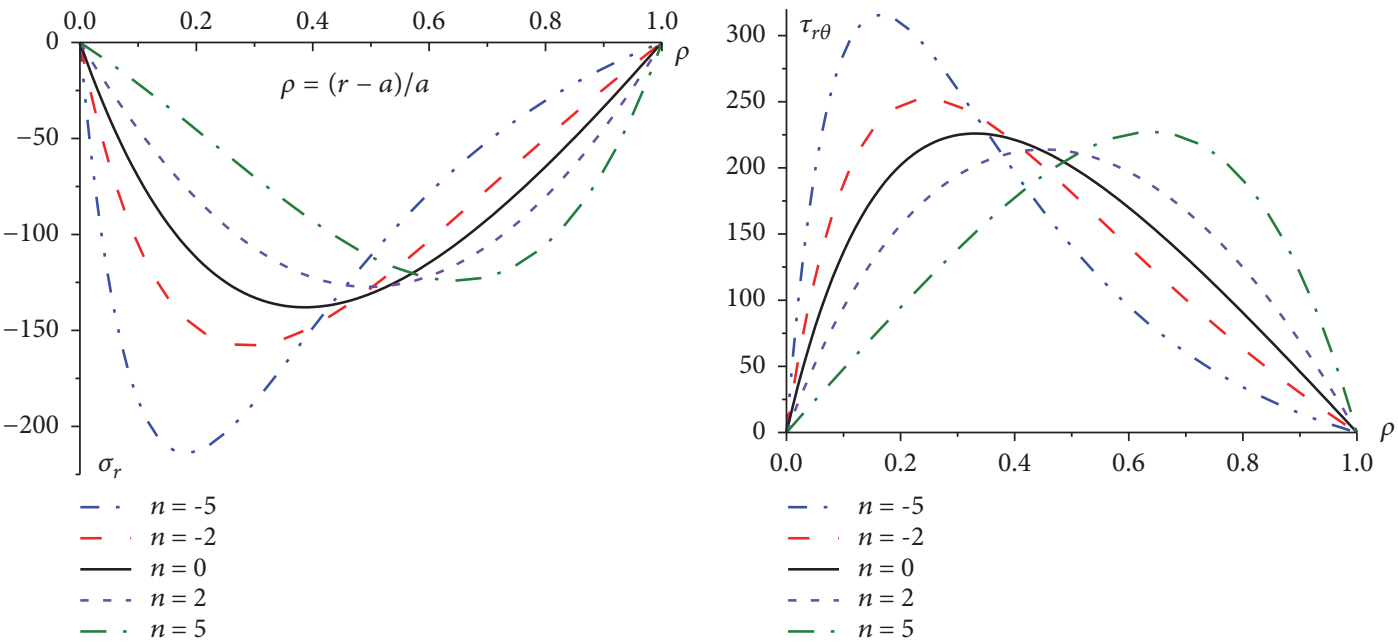

(a)

(b) $\rho=(r-a) / a$
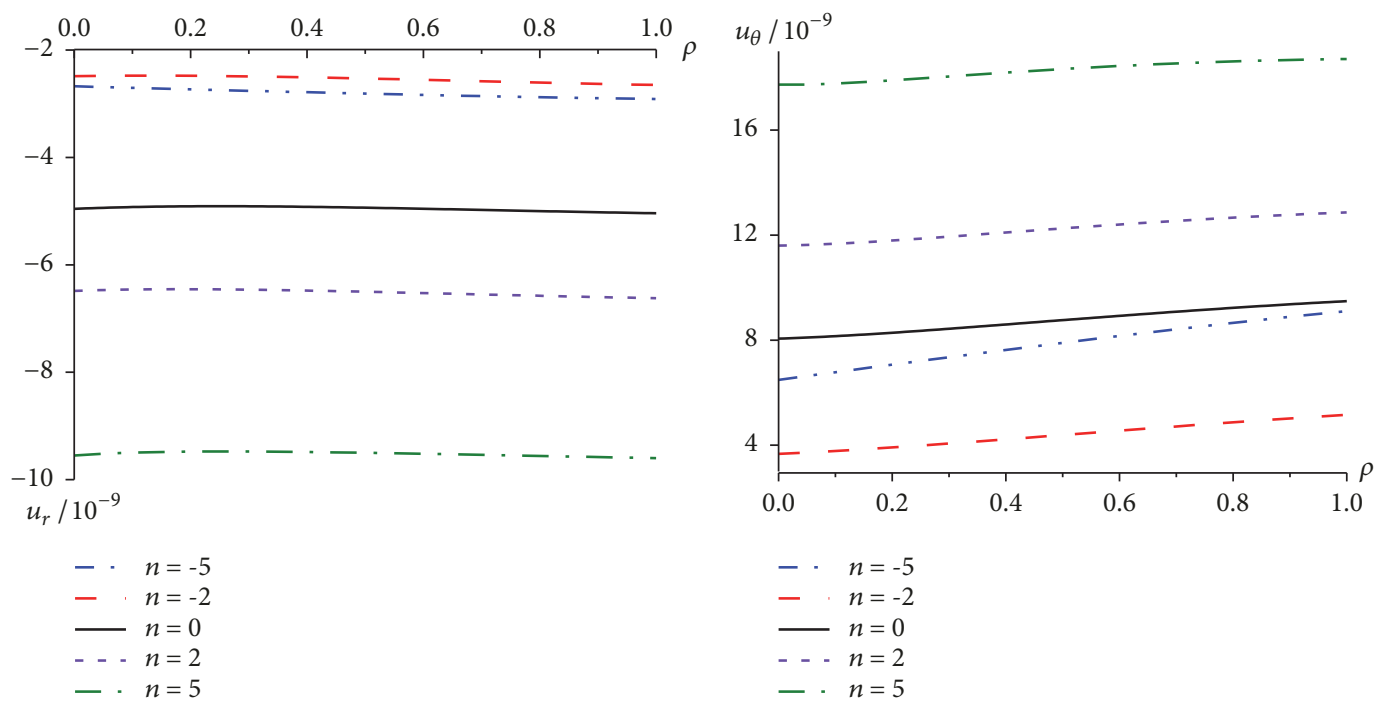

(c) $\rho=(r-a) / a$

(d) $\rho=(r-a) / a$

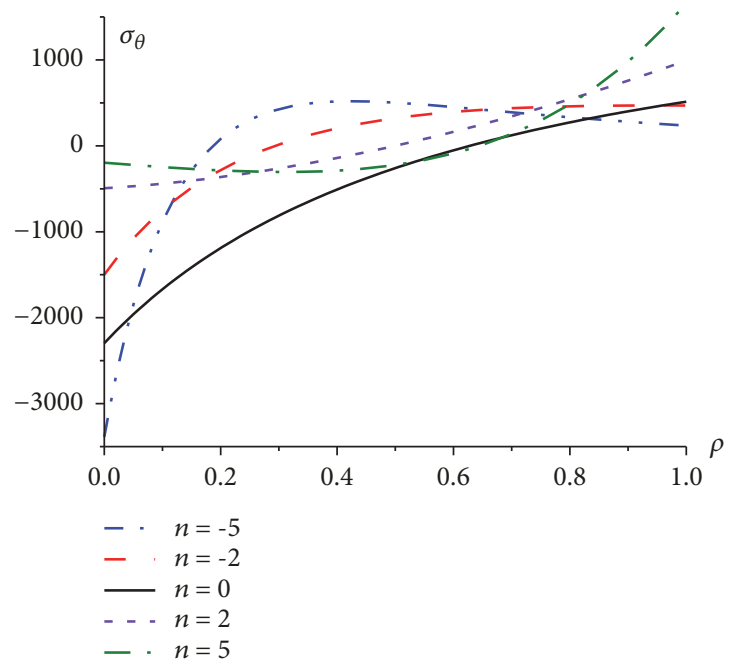

(e) $\rho=(r-a) / a$

FIGURE 4: Variation of physical quantities of a functionally graded curved beam with nondimensional coordinate $\rho$ and a tension force $P$ applied at the free end, when $\theta=\pi / 3$ : (a) stress $\sigma_{r}(\mathrm{~Pa})$; (b) stress $\tau_{r \theta}(\mathrm{Pa})$; (c) displacement $u_{r}(m)$; (d) displacement $u_{\theta}(m)$; (e) stress $\sigma_{\theta}(\mathrm{Pa})$. 


\section{Acknowledgments}

The work is supported by the National Natural Science Foundation of China (Nos. 11472299 and 51704015), China Agricultural University Education Foundation (No. 1101240001).

\section{References}

[1] K. H. Lee, Y. J. Lee, and S. B. Cho, "Characteristics of a transiently propagating crack in functionally graded materials," Journal of Mechanical Science and Technology, vol. 23, no. 5, pp. 1306-1322, 2009.

[2] M. Z. Nejad and G. H. Rahimi, "Deformations and stresses in rotating FGM pressurized thick hollow cylinder under thermal load," Scientific Research and Essays, vol. 4, no. 3, pp. 131-140, 2008.

[3] R. S. Khabbaz, B. D. Manshadi, and A. Abedian, "Nonlinear analysis of FGM plates under pressure loads using the higherorder shear deformation theories," Composite Structures, vol. 89, no. 3, pp. 333-344, 2009.

[4] X. Jin, L. Wu, Y. Sun, and L. Guo, "Microstructure and mechanical properties of $\mathrm{ZrO} 2 / \mathrm{NiCr}$ functionally graded materials," Materials Science and Engineering: A Structural Materials: Properties, Microstructure and Processing, vol. 509, no. 1-2, pp. 63-68, 2009.

[5] G. M. Genin and V. Birman, "Micromechanics and structural response of functionally graded, particulate-matrix, fiberreinforced composites," International Journal of Solids and Structures, vol. 46, no. 10, pp. 2136-2150, 2009.

[6] B. V. Sankar, "An elasticity solution for functionally graded beams," Composites Science and Technology, vol. 61, no. 5, pp. 689-696, 2001.

[7] N. Tutuncu and O. Murat, "Exact solutions for stresses in functionally graded pressure vessels," Composites Part B: Engineering, vol. 32, no. 8, pp. 683-686, 2001.

[8] M. Jabbari, S. Sohrabpour, and M. R. Eslami, "Mechanical and thermal stresses in a functionally graded hollow cylinder due to radially symmetric loads," International Journal of Pressure Vessels and Piping, vol. 79, no. 7, pp. 493-497, 2002.

[9] J. Ying, C. F. Lü, and W. Q. Chen, "Two-dimensional elasticity solutions for functionally graded beams resting on elastic foundations," Composite Structures, vol. 84, no. 3, pp. 209-219, 2008.

[10] X.-F. Li, "A unified approach for analyzing static and dynamic behaviors of functionally graded Timoshenko and EulerBernoulli beams," Journal of Sound and Vibration, vol. 318, no. 4-5, pp. 1210-1229, 2008.

[11] M. Şimşek and T. Kocatürk, "Free and forced vibration of a functionally graded beam subjected to a concentrated moving harmonic load," Composite Structures, vol. 90, no. 4, pp. 465473, 2009.

[12] F. Z. Jouneghani, R. Dimitri, M. Bacciocchi, and F. Tornabene, "Free vibration analysis of functionally graded porous doublycurved shells based on the first-order shear deformation theory," Applied Sciences, vol. 7, no. 12, p. 1252, 2017.

[13] J. Sanchez-Hubert and E. Sanchez Palencia, "Statics of curved rods on account of torsion and flexion," European Journal of Mechanics - A/Solids, vol. 18, no. 3, pp. 365-390, 1999.

[14] N. Hu, B. Hu, B. Yan, H. Fukunaga, and H. Sekine, "Two kinds of C0-type elements for buckling analysis of thin-walled curved beams," Computer Methods Applied Mechanics and Engineering, vol. 171, no. 1-2, pp. 87-108, 1999.

[15] P. Dadras, "Plane strain elastic-plastic bending of a strainhardening curved beam," International Journal of Mechanical Sciences, vol. 43, no. 1, pp. 39-56, 2001.

[16] C. P. Filipich and M. T. Piovan, "The dynamics of thick curved beams constructed with functionally graded materials," Mechanics Research Communications, vol. 37, no. 6, pp. 565-570, 2010.

[17] J. T. Oden and A. R. Eugene, Mechanics of Elastic Structures, Hemisphere Publishing Corporation, New York, NY, USA, 1981.

[18] S. P. Timoshenko, "LXVI. On the correction for shear of the differential equation for transverse vibrations of prismatic bars," The London, Edinburgh, and Dublin Philosophical Magazine and Journal of Science, vol. 41, no. 245, pp. 744-746, 1921.

[19] S. P. Timoshenko, "On the transverse vibrations of bars of uniform cross-section," The London, Edinburgh, and Dublin Philosophical Magazine and Journal of Science, vol. 43, no. 253, pp. 125-131, 1922.

[20] C. W. Lim, C. M. Wang, and S. Kitipornchai, "Timoshenko curved beam bending solutions in terms of Euler-Bernoulli solutions," Archive of Applied Mechanics, vol. 67, no. 3, pp. 179190, 1997.

[21] J. Lee, "In-plane free vibration analysis of curved timoshenko beams by the pseudospectral method," KSME International Journal, vol. 17, no. 8, pp. 1156-1163, 2003.

[22] A. Lyckegaard and O. T. Thomsen, "Nonlinear analysis of a curved sandwich beam joined with a straight sandwich beam," Composites Part B: Engineering, vol. 37, no. 2-3, pp. 101-107, 2005.

[23] S. P. Timoshenko and J. N. Goodier, Theory of Elasticity, McGraw-Hill, New York, NY, USA, 1970.

[24] O. Kiliç and A. Aktaş, "Determination of stress functions of a curved beam subjected to an arbitrarily directed single force at the free end," Mathematical and Computational Applications, vol. 7, no. 2, pp. 181-188, 2002.

[25] C. Bagci, "Exact elasticity solutions for stresses and deflections in curved beams and rings of exponential and t-sections," Journal of Mechanical Design, vol. 115, no. 3, pp. 346-358, 1993.

[26] S. G. Lekhnitskii, Theory of Elasticity of An Anisotropic Body, Mir Publishers, Moscow, Russia, 1981.

[27] J. Dryden, "Bending of inhomogeneous curved bars," International Journal of Solids and Structures, vol. 44, no. 11-12, pp. 4158-4166, 2007.

[28] M. Wang and Y. Liu, "Elasticity solutions for orthotropic functionally graded curved beams," European Journal of Mechanics - A/Solids, vol. 37, pp. 8-16, 2013.

[29] A. Pydah and A. Sabale, "Static analysis of bi-directional functionally graded curved beams," Composite Structures, vol. 160, pp. 867-876, 2017.

[30] Z. Shi, "Bending behavior of piezoelectric curved actuator," Smart Materials and Structures, vol. 14, no. 4, pp. 835-842, 2005.

[31] Z. Shi and T. Zhang, "Bending analysis of a piezoelectric curved actuator with a generally graded property for the piezoelectric parameter," Smart Materials and Structures, vol. 17, no. 4, Article ID 045018, 2008.

[32] A. G. de Miguel, G. De Pietro, E. Carrera, G. Giunta, and A. Pagani, "Locking-free curved elements with refined kinematics for the analysis of composite structures," Computer Methods Applied Mechanics and Engineering, vol. 337, pp. 481-500, 2018.

[33] G. De Pietro, A. G. de Miguel, E. Carrera, G. Giunta, S. Belouettar, and A. Pagani, "Strong and weak form solutions of 
curved beams via Carrera's unified formulation," Mechanics of Advanced Materials and Structures, pp. 1-12, 2018.

[34] L. Y. Yu and L. L. Zhang, "Bending solution of functionally graded curved-beam," Journal of Engineering Mechanics, vol. 31, no. 12, pp. 4-10, 2014. 


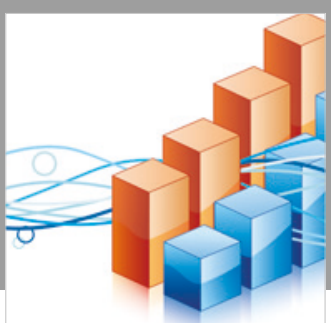

Advances in

Operations Research

\section{-n-m}
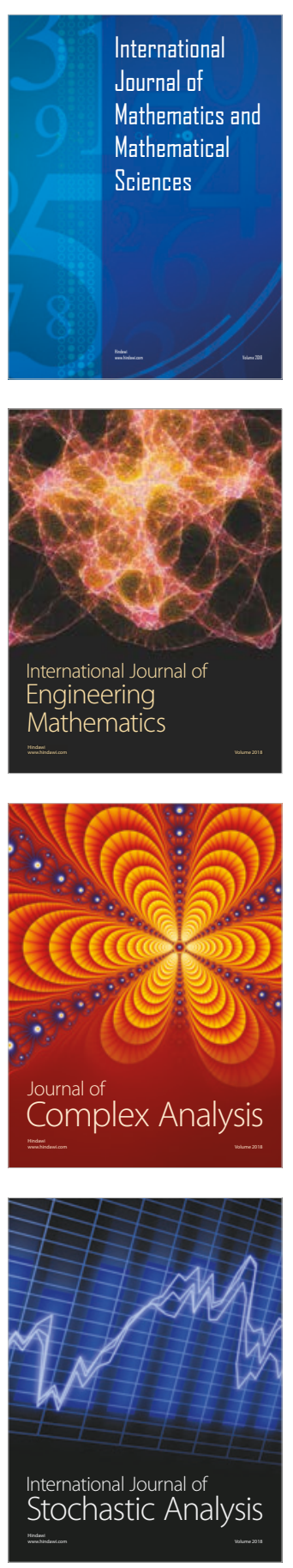
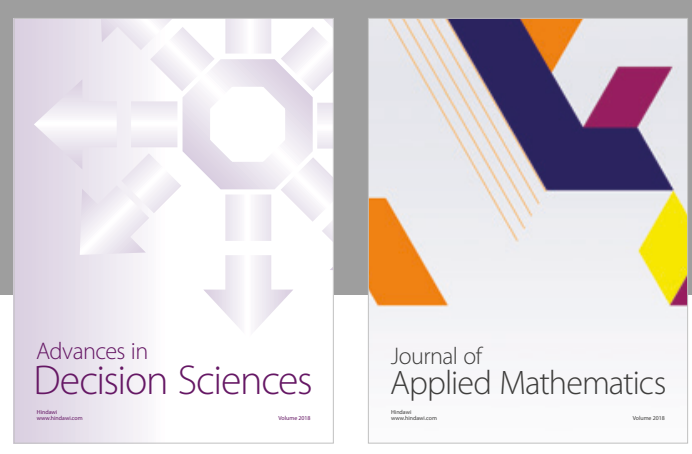

Journal of

Applied Mathematics
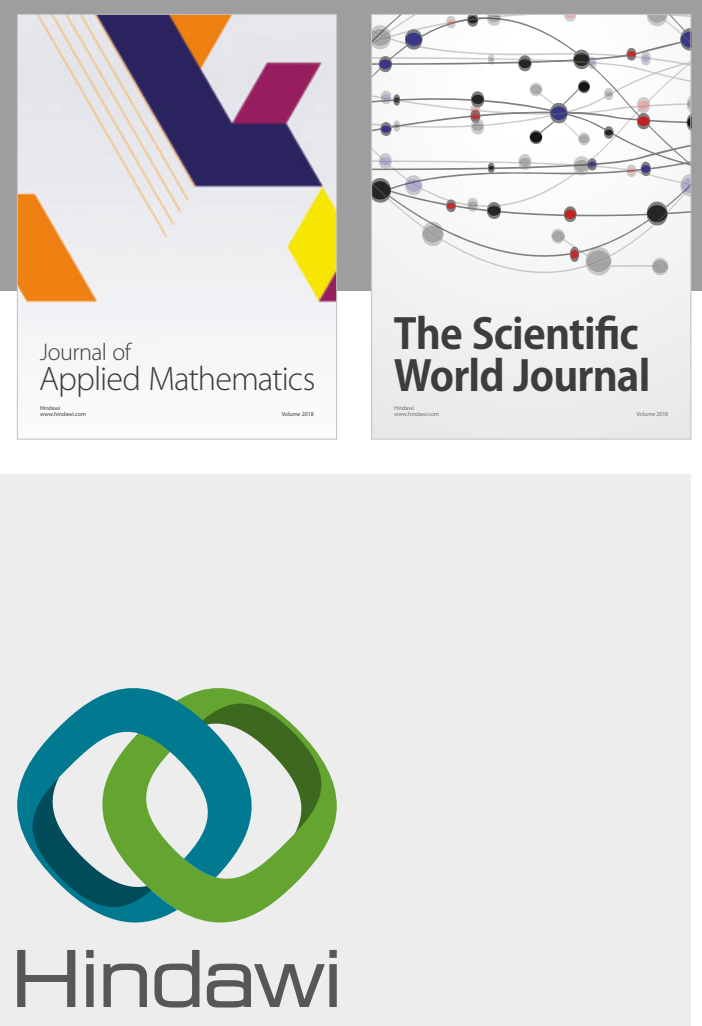

Submit your manuscripts at

www.hindawi.com

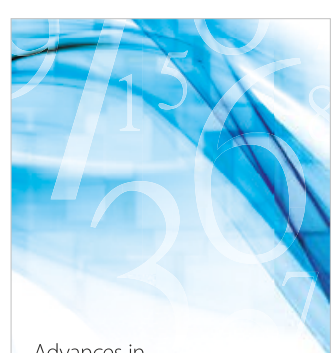

Advances in
Numerical Analysis
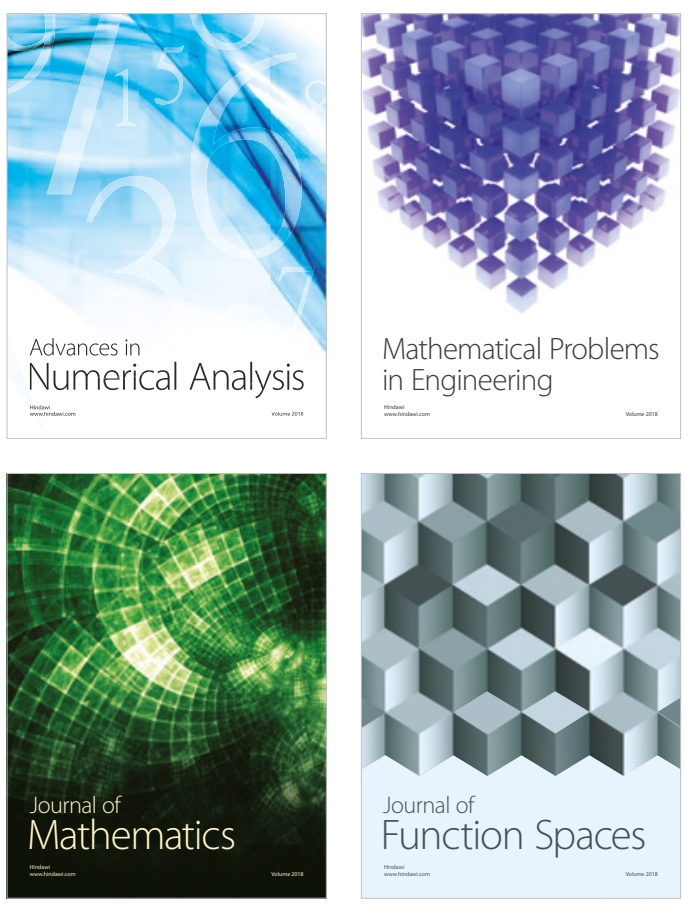

Mathematical Problems in Engineering

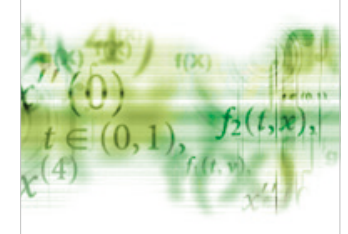

International Journal of

Differential Equations

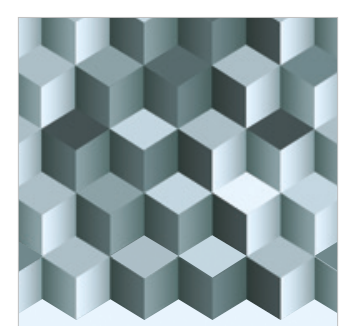

Journal of

Function Spaces

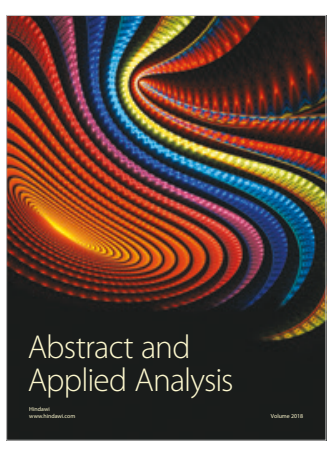

The Scientific

World Journal

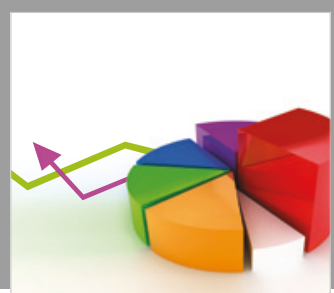

Journal of

Probability and Statistics
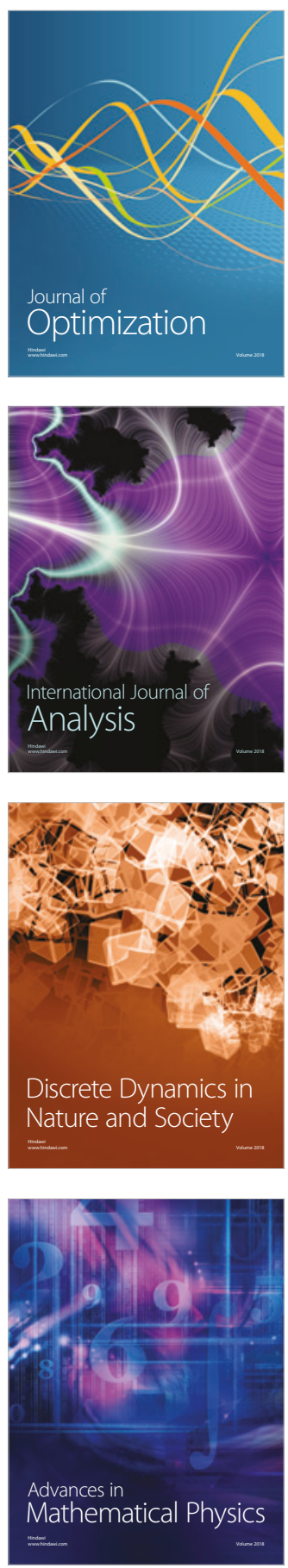